\title{
The Feuerbach Theorem and Cyclography in Universal Geometry
}

\section{The Feuerbach Theorem and Cyclography in Uni- versal Geometry}

\section{ABSTRACT}

We have another look at the Feuerbach theorem with a view to extending it in an oriented way to finite fields using the purely algebraic approach of rational trigonometry and universal geometry. Our approach starts with the tangent lines to three rational points on the unit circle, and all subsequent formulas involve the three parameters that define them. Tangency of incircles is treated in the oriented setting via a simplified form of cyclography. Some interesting features of the finite field case are discussed.

Key words: Feuerbach theorem, incircles, universal geometry, cyclography, finite fields

MSC2010: 51N10, 51E26, 01A55

\section{Introduction}

This paper looks to show that the Feuerbach Theorem, on the tangency of the nine-point circle of a triangle with the four incircles/excircles, holds for triangle geometry over a general field, once the existence of an incircle is established. While such an assumption is implicit in many geometric constructions, algebraically it requires a solution to a quadratic equation, involving number theoretic conditions. This is because incentres rely on bilines of a triangle, which are the rational equivalents of angle bisectors in Rational Trigonometry ([9], [10]). Note also that we adopt the four-fold symmetry towards the incentre hierarchy of [6], where all four incentres /excentres are just referred to as incentres. Over a general field, there is not a good distinction between these.

Our approach starts with the unit circle as an incircle, choosing three rational points on it with parameters $t_{1}, t_{2}$

Feuerbachov teorem i ciklografija u univerzalnoj geometriji

\section{SAŽETAK}

Dajemo drugačiji pogled na Feuerbachov teorem s ciljem da ga se orijentirano proširi na konačna polja koristeći isključivo algebarski pristup racionalne trigonometrije $\mathrm{i}$ univerzalne geometrije. Naš pristup počinje s tangentama u tri racionalne točke na jediničnoj kružnici, i sve naknadne formule uključuju tri parametra koja ih definiraju. Tangencijalnost upisanih kružnica promatra se $u$ orijentiranom okruženju koristeći pojednostavljene forme ciklografije. Promatraju se neka zanimljiva događanja u slučaju konačnih polja.

Ključne riječi: Feuerbachov teorem, upisane kružnice, univerzalna geometrija, ciklografija, konačna polja

and $t_{3}$, and then creating the basic starting triangle via the tangents to these points. We are then able to make numerous calculations of important points and lines in terms of $t_{1}, t_{2}$ and $t_{3}$, culminating with the novel result that all four incentre quadrances are indeed squares in the underlying field.

This then allows us to apply a simple form of the classical theory of cyclography, which connects oriented_circles to relativistic geometry, even in the context of general fields, and which yields a purely algebraic proof of the Feuerbach theorem. So our approach establishes the result even over finite fields.

Metrical geometry over finite fields is a subject still largely in its infancy, and this result allows us to investigate several novel features. One of them is what we might call overlapping: a given finite field might actually be too small to support distinct objects that may be familiar in the rational, or more generally unbounded, field situation. To gain 
some intuition in this direction, we examine some discrete versions of the Feuerbach result in some detail over the prime field $\mathbb{F}_{17}$ to illustrate that new phenomenon really do appear in the finite field case. We end with an example in $\mathbb{F}_{23}$ which contains all the points of the Feuerbach configuration without duplication.

\section{History of the Feuerbach theorem}

Recall that the nine-point circle of a triangle passes through the three midpoints of the sides, the three feet of the altitudes, and the three midpoints between the vertices and the orthocentre.

Theorem 1 (Feuerbach) The nine-point circle of a triangle is tangent to the four incircles of that triangle.

This celebrated theorem is one of the most challenging results in classical geometry, as its statement is simple and surprising, but proofs are highly non-trivial. It was published in 1822 by Karl Wilhelm Feuerbach in the book "Eigenschaften einiger merkwürdigen Punkte des geradlinigen Dreiecks und mehrerer durch sie bestimmten Linien und Figuren. Eine analytisch-trigonometrische Abhandlung" ([3]). Since Feuerbach's initial exposition, many authors have attempted proofs. Almost all of them have relied on distances and/or angles, making them difficult to extend to more general situations, for example geometry over finite fields.

John Casey's 1864 proof ([1]) used inversion and a form of Ptolemy's theorem for collinear points, along with a suitable inversion and comparison of distance ratios of the four incircles and the nine-point circle. Somewhat similar proofs appeared in books of William M'Clelland (1891) ([7]) and Robert Lachlan (1893) ([5]) as semi-completed exercises for the reader. However these proofs only covered the tangency of the interior incircle with the ninepoint circle, with the proof for the tangency of the exterior incircles omitted, and relied on arguments involving distances, angles and concyclic quadrangles.

Coxeter and Greitzer's 1967 proof ([2]) builds upon Casey's proof and streamlines it, using a sequence of inversions and applications of Heron's formula to set up and transform different distance ratios to check what constructions can be preserved under a given inversion.

Franz Hofbauer's 2016 proof ([4]) currently stands as perhaps the simplest proof, and uses the same skeleton as Michael Scheer's 2011 proof ([8]), except that where Scheer used barycentric coordinates and the law of sines, Hofbauer uses vector geometry and Heron's formula. Hofbauer's proof starts with a triangle $\overline{A B C}$ with a circumradius $R$ and side lengths $a, b$ and $c$, chosen such that the circumcentre $O$ of the triangle is centred at the origin. By assigning position vectors to the vertices of the triangle, it becomes simple to find the position vectors of the ninepoint centre, interior incentre and an exterior incentre. After establishing some relationships between the distances in this set up, an application of Heron's formula proves the result.

Both the classical and more modern proofs can be critiqued from several directions, especially when we intend to extend the theorem to universal geometry, over general fields. Distances and angles are hard to generalize, constructions based on diagrams can be problematic, and do we have a consistent theory of inversion and cyclic quadrilaterals over general fields? When we examine in some detail some examples in finite fields at the end of this paper, another possible question emerges: how do we actually know that synthetic arguments create points and lines which are distinct? If further constructions require joins or meets of existing points, there are logical questions here that may be hidden in a proof based on a physical construction.

\section{Projective coordinates and quadrances}

We work over a general field, characteristic two excluded. Projective coordinates for points and lines are useful since cross-product of coordinates express both joins of points and meets of lines. So the affine point $A=[a, b]$ will be expressed in projective coordinates as $A=[1: a: b]$, and the line $l$ with equation $r+s x+t y=0$ will be expressed in projective coordinates as $l=(r, s, t)$. Then the incidence between $A$ and $l$ is expressed as the dot product between their respective coordinates being zero, that is $r+s a+t b=0$. Then for distinct affine points $A=\left[a_{1}, a_{2}\right]$ and $B \equiv\left[b_{1}, b_{2}\right]$ we have their join

$$
\begin{aligned}
A_{1} A_{2} & =\left[1: a_{1}: a_{2}\right] \times\left[1: b_{1}: b_{2}\right] \\
& =\left(a_{1} b_{2}-a_{2} b_{1}: a_{2}-b_{2}: b_{1}-a_{1}\right)
\end{aligned}
$$

and similarly for lines $l_{1}: r_{1}+s_{1} x+t_{1} y=0$ and

$l_{2}: r_{2}+s_{2} x+t_{2} y=0$ their meet is

$$
\begin{aligned}
l_{1} l_{2} & =\left(r_{1}: s_{1}: t_{1}\right) \times\left(r_{2}: s_{2}: t_{2}\right) \\
& =\left[s_{1} t_{2}-s_{2} t_{1}: r_{2} t_{1}-r_{1} t_{2}: r_{1} s_{2}-r_{2} s_{1}\right] .
\end{aligned}
$$

In each case only the usual three-dimensional cross product is involved, thus significantly reducing complexity of elementary calculations.

Using an elementary concept from rational trigonometry ([9]) the (perpendicular) quadrance from a point $P=[a, b]$ to a line $l=(r: s: t)$ with equation $r+s x+t y=0$ is given

$Q(P, l)=\frac{(r+s a+t b)^{2}}{s^{2}+t^{2}}$

assuming the line is non-null, that is its normal vector $(s, t)$ is not null, meaning that $Q((s, t))=s^{2}+t^{2} \neq 0$.

A circle will be an equation of the form $(x-a)^{2}+$ $(y-b)^{2}=Q$, with centre $[a, b]$ and quadrance $Q$. We do 
not assume that $Q$ is necessarily a square in the field, so in general a circle does not have a well-defined "radius". When it does, then there will be two possible associated radii. This observation will play an important role when we discuss cyclography later.

\section{Rational points on the unit circle}

Since all starting incircles are equivalent under translation and dilation, (actually the latter might require a quadratic field extension) we can, without loss of generality, start with the unit circle $C_{0}: x^{2}+y^{2}=1$ with centre $I_{0}=[0,0]$ and quadrance $R_{0}=1$ which we will arrange to be an incircle of our basic triangle. We choose three distinct points on the unit circle

$Z_{i}=\left[1+t_{i}^{2}: 1-t_{i}^{2}: 2 t_{i}\right]=\left[\frac{1-t_{i}^{2}}{1+t_{i}^{2}}, \frac{2 t_{i}}{1+t_{i}^{2}}\right]$ for $i=1,2,3$

where each $t$ value in the field is distinct. The geometrical meaning of the parameter values is shown in Figure 1.

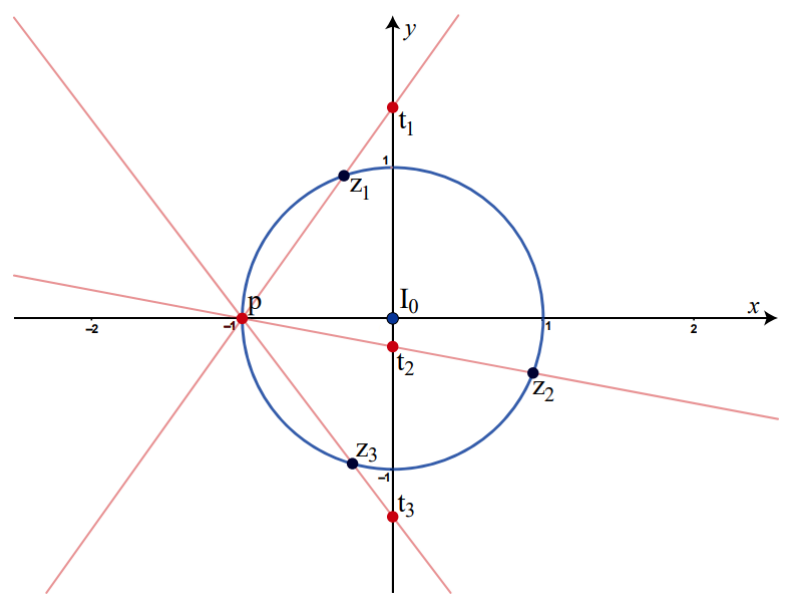

Figure 1: Parameters $t$ correspond to y-intercepts

Remark 1 In order to guarantee the existence of the points on the circle, our choice of t-values must be such that $t^{2}+1 \neq 0$. For the rational numbers, and a prime field $F_{p}$ satisfying $p \equiv 3(\bmod 4)$ this condition holds automatically, but not so for a finite prime field where $p \equiv 1$ $(\bmod 4)$.

Since the tangent to the point $[r, s]$ on the unit circle has equation $r x+s y=1$, with projective coordinates $(-1: r: s)$, the tangent to $C_{0}$ at $Z_{i}$ will be the line $z_{i}=\left(-\left(1+t_{i}^{2}\right): 1-t_{i}^{2}: 2 t_{i}\right)$ for $i=1,2,3$.

We can then define the vertex $A_{1}$ to be the meet of $z_{2}$ and $z_{3}$, and similarly define $A_{2}$ and $A_{3}$ which gives us our basic triangle $\overline{A_{1} A_{2} A_{3}}$ which a calculation shows has vertices

$$
\begin{aligned}
& A_{1} \equiv z_{2} z_{3}=\left[1+t_{2} t_{3}, 1-t_{2} t_{3}, t_{2}+t_{3}\right] \\
& A_{2} \equiv z_{3} z_{1}=\left[1+t_{3} t_{1}, 1-t_{3} t_{1}, t_{3}+t_{1}\right] \\
& A_{3} \equiv z_{1} z_{2}=\left[1+t_{1} t_{2}, 1-t_{1} t_{2}, t_{1}+t_{2}\right] .
\end{aligned}
$$

To avoid having any vertices at infinity, we will further require that no two $t$-values have a product of -1 , since tangents $z_{i}$ and $z_{j}$ are parallel precisely when the chosen points $Z_{i}$ and $Z_{j}$ are diametrically opposed, which amounts to the condition $t_{i} t_{j}=-1$. This construction guarantees that the unit circle $C_{0}$ will be tangent to the three sides of the triangle $\overline{A_{1} A_{2} A_{3}}$, meaning it is one of the incircles of the triangle.

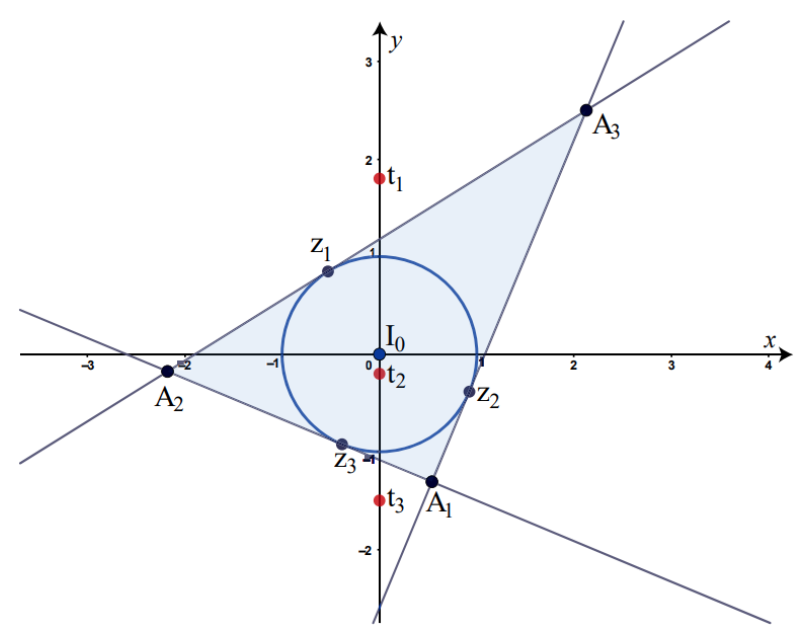

Figure 2: If $t_{1}=1.8, t_{2}=-0.2$ and $t_{3}=-1.5, C_{0}$ is the interior incircle

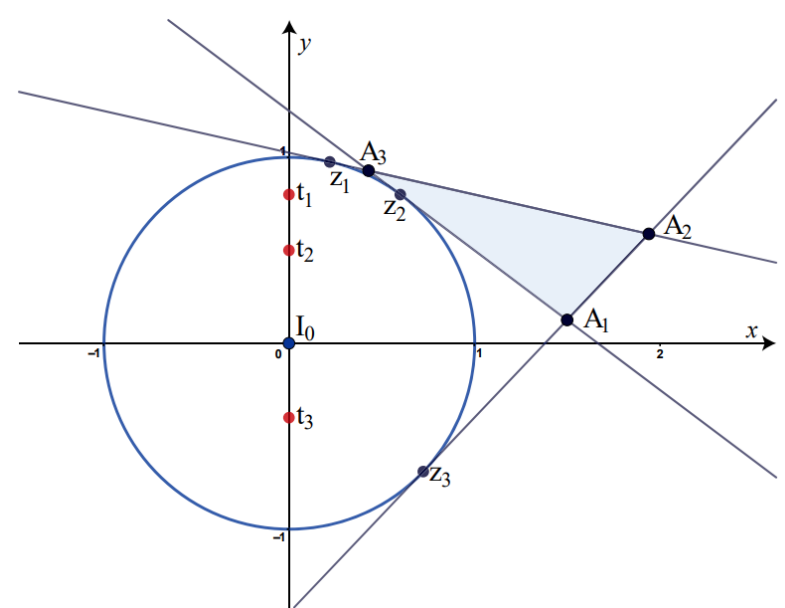

Figure 3: If $t_{1}=0.8, t_{2}=0.5$ and $t_{3}=-0.4, C_{0}$ is an exterior incircle

Remark 2 Over the rational numbers, different choices for the $t$-values will determine whether or not $C_{0}$ will be the interior incircle or one of the exterior incircles. Indeed, it can be shown that $C_{0}$ will be the interior incircle 
precisely when $\left(t_{1} t_{2}+1\right)\left(t_{1} t_{3}+1\right)\left(t_{2} t_{3}+1\right)<0$. But over general fields this kind of consideration is reduced in importance.

\section{The incentres}

Since we know that $I_{0}$ is an incentre, it is determined as the meet of three of the bilines of the triangle $\overline{A_{1} A_{2} A_{3}}$, with one biline from each vertex. The biline $b_{1 a}$ is the join of $I_{0}=[0,0]$ and $A_{1}$ so in projective coordinates it is

$$
\begin{aligned}
b_{1 a} & =[1: 0: 0] \times\left[1+t_{2} t_{3}: 1-t_{2} t_{3}: t_{2}+t_{3}\right] \\
& =\left(0:-\left(t_{2}+t_{3}\right): 1-t_{2} t_{3}\right)
\end{aligned}
$$

and similarly

$b_{2 a}=\left(0:-\left(t_{3}+t_{1}\right): 1-t_{3} t_{1}\right)$

and

$b_{3 a}=\left(0:-\left(t_{1}+t_{2}\right): 1-t_{1} t_{2}\right)$.

Since bilines come in pairs, meeting perpendicularly at their corresponding vertex, we can use the above equations to find the remaining three bilines of $\overline{A_{1} A_{2} A_{3}}$. For example: the biline perpendicular to $b_{1 a}$ at $A_{1}$ will have equation

$b_{1 b}:\left(1-t_{2} t_{3}\right) x+\left(t_{2}+t_{3}\right) y=c$

and substituting the coordinates of $A_{1}$ gives $\frac{\left(1-t_{2} t_{3}\right)^{2}}{1+t_{2} t_{3}}+$ $\frac{\left(t_{2}+t_{3}\right)^{2}}{1+t_{2} t_{3}}=c$ which simplifies to $c=\frac{\left(1+t_{2}^{2}\right)\left(1+t_{3}^{2}\right)}{\left(1+t_{2} t_{3}\right)}$. Doing this for the pairs of bilines corresponding to each vertex yields the projective coordinates of the remaining bilines:

$b_{1 b}=$

$\left(-\left(1+t_{2}^{2}\right)\left(1+t_{3}^{2}\right):\left(1-t_{2} t_{3}\right)\left(1+t_{2} t_{3}\right):\left(t_{2}+t_{3}\right)\left(1+t_{2} t_{3}\right)\right)$

$b_{2 b}=$

$\left(-\left(1+t_{3}^{2}\right)\left(1+t_{1}^{2}\right):\left(1-t_{3} t_{1}\right)\left(1+t_{3} t_{1}\right):\left(t_{3}+t_{1}\right)\left(1+t_{3} t_{1}\right)\right)$

$b_{3 b}=$

$\left(-\left(1+t_{1}^{2}\right)\left(1+t_{2}^{2}\right):\left(1-t_{1} t_{2}\right)\left(1+t_{1} t_{2}\right):\left(t_{1}+t_{2}\right)\left(1+t_{1} t_{2}\right)\right)$.

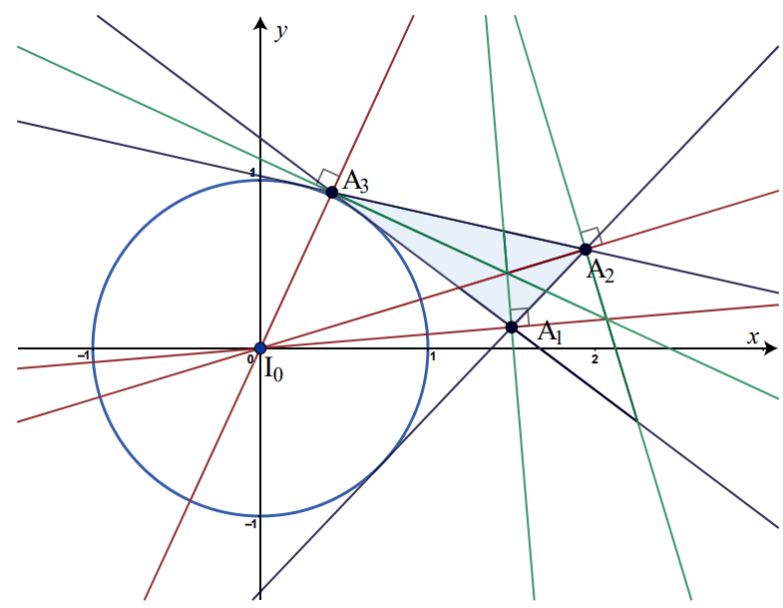

Figure 4: All six bilines of $\overline{A_{1} A_{2} A_{3}}$

These six bilines meet three at a time at the four incentres, which besides $I_{0}=[0,0]$ are

$I_{1} \equiv b_{2 b} b_{3 b}=$

$\left[\left(1+t_{1} t_{2}\right)\left(1+t_{3} t_{1}\right):\left(1+t_{1}^{2}\right)\left(1-t_{2} t_{3}\right):\left(1+t_{1}^{2}\right)\left(t_{2}+t_{3}\right)\right]$

$I_{2} \equiv b_{1 b} b_{3 b}=$

$\left[\left(1+t_{1} t_{2}\right)\left(1+t_{2} t_{3}\right):\left(1+t_{2}^{2}\right)\left(1-t_{3} t_{1}\right):\left(1+t_{2}^{2}\right)\left(t_{3}+t_{1}\right)\right]$

$I_{3} \equiv b_{1 b} b_{2 b}=$

$\left[\left(1+t_{3} t_{1}\right)\left(1+t_{2} t_{3}\right):\left(1+t_{3}^{2}\right)\left(1-t_{1} t_{2}\right):\left(1+t_{3}^{2}\right)\left(t_{1}+t_{2}\right)\right]$.

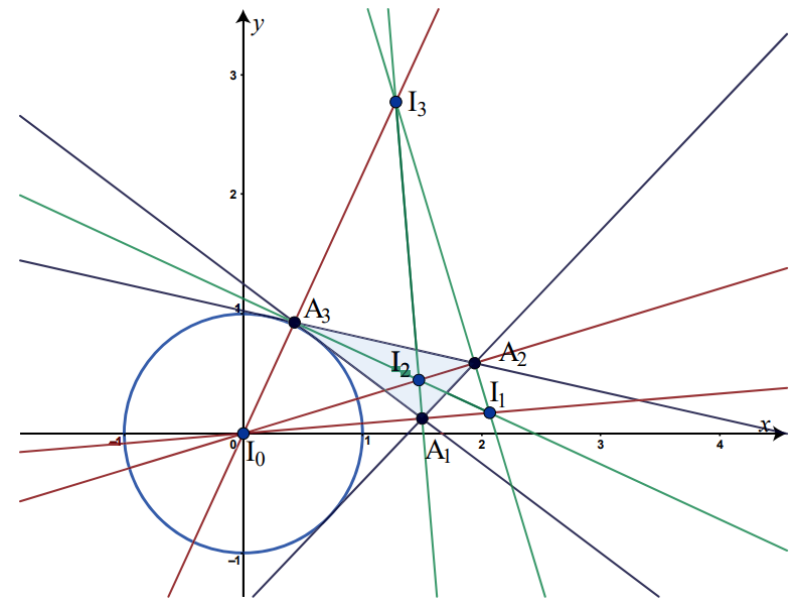

Figure 5: Incentres $I_{0}, I_{1}, I_{2}, I_{3}$ of the triangle $\overline{A_{1} A_{2} A_{3}}$

\section{Equations of the incircles}

Now that we have the centres of the incircles, we may determine their quadrances, using the formula for the quad- 
rance from a point to a line, to get

$$
\begin{aligned}
& R_{1} \equiv Q\left(I_{1}, z_{1}\right)=\frac{\left(t_{1}-t_{2}\right)^{2}\left(t_{1}-t_{3}\right)^{2}}{\left(1+t_{1} t_{2}\right)^{2}\left(1+t_{1} t_{3}\right)^{2}} \\
& R_{2} \equiv Q\left(I_{2}, z_{2}\right)=\frac{\left(t_{2}-t_{3}\right)^{2}\left(t_{2}-t_{1}\right)^{2}}{\left(1+t_{1} t_{2}\right)^{2}\left(1+t_{2} t_{3}\right)^{2}} \\
& R_{3} \equiv Q\left(I_{3}, z_{3}\right)=\frac{\left(t_{3}-t_{1}\right)^{2}\left(t_{3}-t_{2}\right)^{2}}{\left(1+t_{1} t_{3}\right)^{2}\left(1+t_{2} t_{3}\right)^{2}} .
\end{aligned}
$$

Remark 3 Since each incentre will be equidistant to the three sides of the triangle $\overline{A_{1} A_{2} A_{3}}$, we could actually use any of the tangents $z_{1}, z_{2}$ or $z_{3}$ to compute the quadrances of the incircles.

As a consequence of these formulas, we may make the following deduction:

Theorem 2 If one of the incircles of a triangle has a quadrance which is a perfect square, then so do the others.

Proof. If one of the incircles has a quadrance which is a square, then we can position and dilate it to be the unit circle using only affine transformations involving the field, in which case the above formulas hold. Then translating back and performing an inverse dilation shows that the original incircles also have square quadrances.

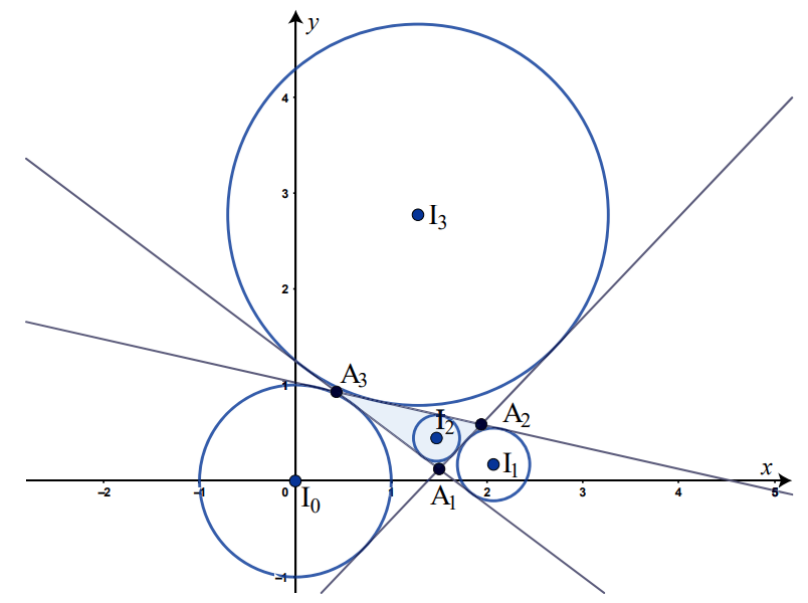

Figure 6: The four incentres and incircles

\section{Coordinates of the nine point centre}

The nine-point circle of a triangle is the circumcircle of the three midpoints of the triangle. The following gives the affine coordinates for the centre $N$ of this nine point circle in terms of the affine coordinates of three general vertices. The formulas are conveniently expressed using determinants of $3 \times 3$ matrices.
Theorem 3 In projective coordinates the centre of the nine-point circle of the triangle $\overline{G_{1} G_{2} G_{3}}$ where $G_{1}=$ $\left[1: a_{1}: b_{1}\right], G_{2}=\left[1: a_{2}: b_{2}\right]$ and $G_{3}=\left[1: a_{3}: b_{3}\right]$, is $N=$ $\left[z_{N}: x_{N}: y_{N}\right]$ where

$z_{N}=4\left|\begin{array}{lll}a_{1} & b_{1} & 1 \\ a_{2} & b_{2} & 1 \\ a_{3} & b_{3} & 1\end{array}\right|$

$x_{N}=\left|\begin{array}{lll}a_{1}^{2} & b_{1} & 1 \\ a_{2}^{2} & b_{2} & 1 \\ a_{3}^{2} & b_{3} & 1\end{array}\right|-2\left|\begin{array}{ccc}a_{1} b_{1} & a_{1} & 1 \\ a_{2} b_{2} & a_{2} & 1 \\ a_{3} b_{3} & a_{3} & 1\end{array}\right|+\left|\begin{array}{ccc}b_{1} & b_{1}^{2} & 1 \\ b_{2} & b_{2}^{2} & 1 \\ b_{3} & b_{3}^{2} & 1\end{array}\right|$

$y_{N}=\left|\begin{array}{lll}b_{1}^{2} & a_{1} & 1 \\ b_{2}^{2} & a_{2} & 1 \\ b_{3}^{2} & a_{3} & 1\end{array}\right|-2\left|\begin{array}{lll}a_{1} b_{1} & b_{1} & 1 \\ a_{2} b_{2} & b_{2} & 1 \\ a_{3} b_{3} & b_{3} & 1\end{array}\right|+\left|\begin{array}{ccc}a_{1} & a_{1}^{2} & 1 \\ a_{2} & a_{2}^{2} & 1 \\ a_{3} & a_{3}^{2} & 1\end{array}\right|$.

Proof. Since the nine-point circle of a triangle is the circumcircle of its median triangle, the nine-point centre is the intersection of the perpendicular bisectors of the median triangle. The median triangle has vertices

$M_{1}=\frac{1}{2} G_{2}+\frac{1}{2} G_{3}=\left[2: a_{2}+a_{3}: b_{2}+b_{3}\right]$

$M_{2}=\frac{1}{2} G_{1}+\frac{1}{2} G_{3}=\left[2: a_{1}+a_{3}: b_{1}+b_{3}\right]$

$M_{3}=\frac{1}{2} G_{1}+\frac{1}{2} G_{2}=\left[2: a_{1}+a_{2}: b_{1}+b_{2}\right]$.

The perpendicular bisector of $M_{1} M_{2}$ has normal vector of $M_{2}-M_{1}=\left(a_{1}-a_{2}, b_{1}-b_{2}\right)$ and passes through the point $\frac{1}{2} M_{1}+\frac{1}{2} M_{2}$ so we can write it as

$$
\begin{array}{r}
{\left[\left(a_{1}-a_{2}, b_{1}-b_{2}\right)\left(\frac{1}{4}\left(a_{1}+a_{2}+2 a_{3}\right), \frac{1}{4}\left(b_{1}+b_{2}+2 b_{3}\right)\right)^{T}:\right.} \\
\left.: a_{1}-a_{2}: b_{1}-b_{2}\right]
\end{array}
$$

and, similarly, the perpendicular bisector of $M_{1} M_{3}$ can be written as

$$
\begin{array}{r}
{\left[\left(a_{1}-a_{3}, b_{1}-b_{3}\right)\left(\frac{1}{4}\left(a_{1}+2 a_{2}+a_{3}\right), \frac{1}{4}\left(b_{1}+2 b_{2}+b_{3}\right)\right)^{T}:\right.} \\
\left.: a_{1}-a_{3}: b_{1}-b_{3}\right] .
\end{array}
$$

The meet of these two lines may then be computed and re-expressed in terms of determinants as above.

Theorem 4 For our basic triangle $\overline{A_{1} A_{2} A_{3}}$ determined by parameters $t_{1}, t_{2}$ and $t_{3}$, the nine-point centre is $N=$ $\left[z_{N}: x_{N}: y_{N}\right]$ where

$$
\begin{aligned}
z_{N}= & 8\left(t_{2} t_{3}+1\right)\left(t_{1} t_{3}+1\right)\left(t_{1} t_{2}+1\right) \\
x_{N}= & -\left(3 t_{1} t_{2} t_{3}+t_{1} t_{2}+t_{1} t_{3}+t_{2} t_{3}+t_{1}+t_{2}+t_{3}+3\right) \\
& \cdot\left(3 t_{1} t_{2} t_{3}-t_{1} t_{2}-t_{1} t_{3}-t_{2} t_{3}+t_{1}+t_{2}+t_{3}-3\right) \\
y_{N}= & 2\left(t_{1}+t_{2}+t_{3}+3 t_{1} t_{2} t_{3}\right)\left(t_{1} t_{2}+t_{1} t_{3}+t_{2} t_{3}+3\right) .
\end{aligned}
$$


Proof. We attain this result by substituting the coordinates of the three points $A_{1}, A_{2}$ and $A_{3}$ into the expressions for the nine-point centre as determined by the previous theorem and simplifying the result.

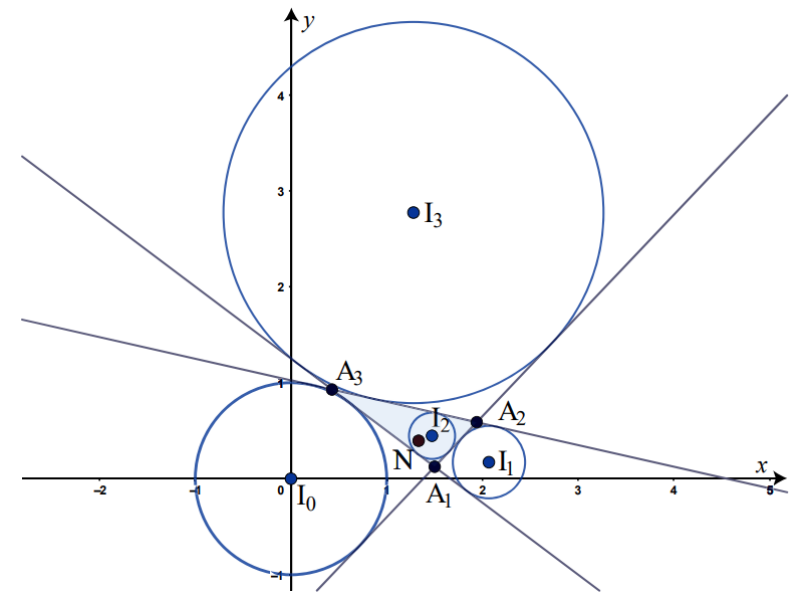

Figure 7: The nine-point centre $N$ of the triangle $\overline{A_{1} A_{2} A_{3}}$

\section{Quadrance of the nine-point circle}

We now come to our first somewhat remarkable calculation, that anticipates the subtlety of the Feuerbach theorem.

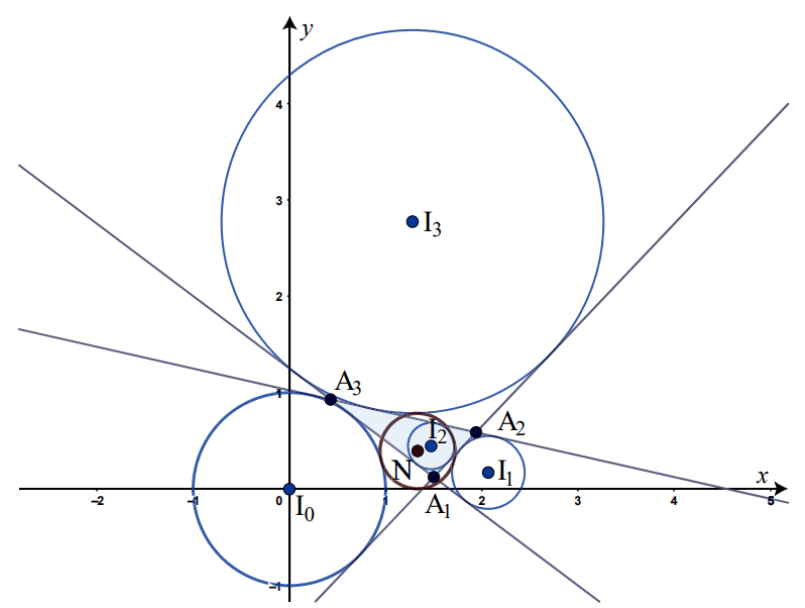

Figure 8: Tangency of the nine-point circle

Theorem 5 The quadrance of the nine-point circle is

$R_{N}=\frac{\left(t_{1}^{2}+1\right)^{2}\left(t_{2}^{2}+1\right)^{2}\left(t_{3}^{2}+1\right)^{2}}{64\left(t_{1} t_{2}+1\right)^{2}\left(t_{1} t_{3}+1\right)^{2}\left(t_{2} t_{3}+1\right)^{2}}$.

Proof. Since the median points of a triangle also lie on the nine-point circle, we can use the nine-point centre and any of the median points of the triangle $\overline{A_{1} A_{2} A_{3}}$ to compute the quadrance of the nine-point circle. A median point of $\overline{A_{1} A_{2} A_{3}}$ is

$$
\begin{aligned}
M_{1} & =\frac{1}{2} A_{2}+\frac{1}{2} A_{3} \\
& =\left[2: \frac{1-t_{1} t_{3}}{1+t_{1} t_{3}}+\frac{1-t_{1} t_{2}}{1+t_{1} t_{2}}: \frac{t_{1}+t_{3}}{1+t_{1} t_{3}}+\frac{t_{1}+t_{2}}{1+t_{1} t_{2}}\right] .
\end{aligned}
$$

So using the formula for the nine-point centre in the previous theorem, and after some miraculous simplification,

$$
\begin{aligned}
R_{N} & =Q\left(I_{N}, M_{1}\right)= \\
& \left(-\frac{\left(3 t_{1} t_{2} t_{3}+t_{1} t_{2}+t_{1} t_{3}+t_{2} t_{3}+t_{2}-t_{1} t_{3}-t_{2} t_{3}+t_{1}+t_{2}+t_{3}-3\right)}{8\left(t_{2} t_{3}+1\right)\left(t_{1} t_{3}+1\right)\left(t_{1} t_{2}+1\right)}\right. \\
& \left.-\frac{\frac{1-t_{1} t_{3}}{1+t_{1} t_{3}}+\frac{1-t_{1} t_{2}}{1+t_{1} t_{2}}}{2}\right)^{2} \\
+ & \frac{\left(t_{1}+t_{2}+t_{3}+3 t_{1} t_{2} t_{3}\right)\left(t_{1} t_{2}+t_{1} t_{3}+t_{2} t_{3}+3\right)}{4\left(t_{2} t_{3}+1\right)\left(t_{1} t_{3}+1\right)\left(t_{1} t_{2}+1\right)} \\
- & \left.\frac{\frac{t_{1}+t_{3}}{1+t_{1} t_{3}}+\frac{t_{1}+t_{2}}{1+t_{1} t_{2}}}{2}\right)^{2} \\
= & \frac{\left(t_{1}^{2}+1\right)^{2}\left(t_{2}^{2}+1\right)^{2}\left(t_{3}^{2}+1\right)^{2}}{64\left(t_{1} t_{2}+1\right)^{2}\left(t_{1} t_{3}+1\right)^{2}\left(t_{2} t_{3}+1\right)^{2}} .
\end{aligned}
$$

Remark 4 Notice that this quadrance for the nine-point circle is also a square.

Our aim is to establish the Feuerbach theorem, but the previous results suggest that we can restate this in terms of the classical theory of oriented cycles, or cyclography.

\section{Cyclography}

We now introduce a very simple version of the 19th century theory of cyclography, but more generally over an arbitrary field $\mathbb{F}$. An oriented_circle (note that we employ the usage of one word) is an affine 3-point $C=[a, b, r]$ in the affine space $\mathbb{A}^{3}$ over the field $\mathbb{F}$. The affine 2-point $P=[a, b]$ in $\mathbb{A}^{2}$ is called the centre of $C$, while the number $r$ is called the oriented_radius of $C$, and we will also write $C=[P, r]$. Two oriented_circles are equal precisely when both their centres and oriented_radii agree. A point $[x, y]$ lies on $C=[a, b, r]$ precisely when

$(a-x)^{2}+(b-y)^{2}=r^{2}$.

This is the equation of a circle $c$ with centre also $[a, b]$ and quadrance $Q=r^{2}$. Clearly both $C=[a, b, r]$ and $C^{\prime}=$ $[a, b,-r]$ are associated to the same circle $c$. Note however that a general circle cannot be expected to have associated oriented_circles: this will happen precisely when its quadrance is a square in $\mathbb{F}$. 
When $r=0$ we say the oriented_circle is a null circle Over the rational numbers such null oriented_circles have only one point lying on them. This gives us the null plane of null circles inside the three-dimensional space of points $[a, b, r]$.

The space of oriented_circles is naturally identified with the three-dimensional affine space $A^{3}$ over $\mathbb{F}$. The geometry of oriented_circles however embues this threedimensional space $\mathbb{A}^{3}$ with a relativistic $2+1$ quadratic form. In the rational case, non-horizontal lines (not in a plane of the form $r=r_{0}$ ) in this space $\mathbb{A}^{3}$ correspond to pencils of homothetic oriented_circles, with the meets of such lines with the plane of null circles giving the (oriented) homothetic centres.

To discuss homothetic centres, let's agree to the useful convention that if $P=[a, b, c]$ is an affine point and $v=$ $[r, s, t]$ is a vector, then $P+v$ is the affine point $P+v=$ $[a+r, b+s, c+t]$. Then for $\alpha \neq 0$, the dilation centred at $P$ by $\alpha$ is the bijection of the plane that sends $P+v$ to $P+\alpha v$. The inverse is clearly the dilation centred at $P$ by $\alpha^{-1}$.

Proposition 1 The unique homothetic centre for the oriented_circles $C_{1}=\left[a_{1}, b_{1}, r_{1}\right]$ and $C_{2}=\left[a_{2}, b_{2}, r_{2}\right]$ where $r_{1} \neq r_{2}$ is

$P=\left[\frac{a_{1} r_{2}-a_{2} r_{1}}{r_{2}-r_{1}}, \frac{b_{1} r_{2}-b_{2} r_{1}}{r_{2}-r_{1}}\right]$.

If $r_{1} \neq 0$ then the dilation in the plane with centre $P$ by $a$ factor of $r_{2} / r_{1}$ sends $C_{1}$ to $C_{2}$, and if $r_{2} \neq 0$ then the plane dilation with centre $P$ by a factor of $r_{1} / r_{2}$ sends $C_{2}$ to $C_{1}$.

Proof. If $C_{1}=\left[a_{1}, b_{1}, r_{1}\right]$ and $C_{2}=\left[a_{2}, b_{2}, r_{2}\right]$ are circles with $r_{1} \neq r_{2}$, then the vector $w=\left(a_{2}-a_{1}, b_{2}-b_{1}, r_{2}-r_{1}\right)$ is not horizontal, and so the line through those points of the parametric form

$\left[a_{1}, b_{1}, r_{1}\right]+\lambda w$

will meet the null plane when $r_{1}+\lambda\left(r_{2}-r_{1}\right)=0$. This occurs precisely when $\lambda=-\frac{r_{1}}{r_{2}-r_{1}}$ and gives the point $[P, 0]$ where $P$ is the homothetic centre

$P=\left[\frac{1}{r_{2}-r_{1}}\left(a_{1} r_{2}-a_{2} r_{1}\right), \frac{1}{r_{2}-r_{1}}\left(b_{1} r_{2}-b_{2} r_{1}\right)\right]$.

Thus we can write

$$
\begin{aligned}
& {\left[a_{1}, b_{1}, r_{1}\right]=[P, 0]+\frac{r_{1}}{r_{2}-r_{1}} w \quad \text { and }} \\
& {\left[a_{2}, b_{2}, r_{2}\right]=[P, 0]+\frac{r_{2}}{r_{2}-r_{1}} w}
\end{aligned}
$$

which proves that if $r_{1} \neq 0$ then the plane dilation centred at $P$ by $r_{2} / r_{1}$ sends $C_{1}$ to $C_{2}$, and similarly if $r_{2} \neq 0$ then the dilation by $r_{1} / r_{2}$ sends $C_{2}$ to $C_{1}$.

A point $A_{1}$ lying on $C_{1}$ and a point $A_{2}$ lying on $C_{2}$ are homologous if they are images of each other under these dilations. Now a dilation is an affine map, so directions are maintained, in the sense that the vector determined by the image of two points will be a multiple of the vector determined by the two points themselves. It follows that if $A_{1}$ lying on $C_{1}$ and a point $A_{2}$ lying on $C_{2}$ are homologous, then the vector $\overrightarrow{C_{1} A_{1}}$ will be a multiple of the vector $\overrightarrow{C_{2} A_{2}}$. This yields a direct construction of the homologous point $A_{2}$ to $A_{1}$ once the homothetic centre is determined.

Three points in $\mathbb{F}^{3}$ generally determine a plane, and the meet of such with the null plane will be a line, giving an easy proof of a theorem of Monge that the three homothetic centres of three oriented circles are in general collinear. However if we start with ordinary, unoriented circles that have square quadrances, then due to the choices of possible orientations there will be determined four such Monge lines.

Definition 1 The quadrance between oriented_circles $C_{1}=\left[a_{1}, b_{1}, r_{1}\right]$ and $C_{2}=\left[a_{2}, b_{2}, r_{2}\right]$ is

$Q\left(C_{1}, C_{2}\right) \equiv\left(a_{2}-a_{1}\right)^{2}+\left(b_{2}-b_{1}\right)^{2}-\left(r_{2}-r_{1}\right)^{2}$.

In the case of disjoint oriented_circles in the rational number plane which are not contained in each other, this quadrance has the interpretation of the quadrance along a common homothetic tangent, meaning a tangent which passes through the homothetic centre.

Definition 2 The oriented_circles $C_{1}=\left[a_{1}, b_{1}, r_{1}\right]$ and $C_{2}=\left[a_{2}, b_{2}, r_{2}\right]$ are tangent precisely when $r_{1} \neq r_{2}$ and $Q\left(C_{1}, C_{2}\right)=0$.

In other words, when the quadrance between their centres is equal to the square of this (non-zero) difference of their oriented_radii, that is when

$\left(a_{2}-a_{1}\right)^{2}+\left(b_{2}-b_{1}\right)^{2}=\left(r_{2}-r_{1}\right)^{2}$.

Example 1 The oriented_circles $C=[0,0,1]$ and $D=$ $[2,0,1]$ are not tangent, even though their associated circles are. However the oriented_circles $C=[0,0,1]$ and $E=[2,0,-1]$ are tangent.

It is easy to see that if two oriented_circles are tangent, then so are their associated circles. One of the advantages of the cyclographic set-up is that the points of tangency for oriented circles can be obtained very directly. 
Theorem 6 (Oriented tangency) If the oriented_circles $C_{1}=\left[a_{1}, b_{1}, r_{1}\right]$ and $C_{2}=\left[a_{2}, b_{2}, r_{2}\right]$ are tangent then they meet at exactly one point, which is

$$
\begin{aligned}
J & =\frac{r_{2}}{r_{2}-r_{1}}\left[a_{1}, b_{1}\right]-\frac{r_{1}}{r_{2}-r_{1}}\left[a_{2}, b_{2}\right] \\
& =\left[r_{2}-r_{1}: r_{2} a_{1}-r_{1} a_{2}: r_{2} b_{1}-r_{1} b_{2}\right] .
\end{aligned}
$$

Proof. If the two oriented_circles meet tangentially, they will do so on the line joining their centres, so we can consider a general affine combination

$$
\begin{aligned}
J & =(1-t)\left[a_{1}, b_{1}\right]+t\left[a_{2}, b_{2}\right] \\
& =\left[t a_{2}-a_{1}(t-1), t b_{2}-b_{1}(t-1)\right] .
\end{aligned}
$$

Now in order for this to lie on $C_{1}$ we require that

$\left(t a_{2}-a_{1}(t-1)-a_{1}\right)^{2}+\left(t b_{2}-b_{1}(t-1)-b_{1}\right)^{2}=r_{1}^{2}$

which is just

$\left(\left(a_{2}-a_{1}\right)^{2}+\left(b_{2}-b_{1}\right)^{2}\right) t^{2}=r_{1}^{2}$.

But given that the two oriented_circles are tangent, we can rewrite this as

$\left(r_{2}-r_{1}\right)^{2} t^{2}=r_{1}^{2}$

so that

$t= \pm \frac{r_{1}}{r_{2}-r_{1}}$

Similarly the condition that $J$ lies on $C_{1}$ is

$\left(t a_{2}-a_{1}(t-1)-a_{2}\right)^{2}+\left(t b_{2}-b_{1}(t-1)-b_{2}\right)^{2}=r_{2}^{2}$

or

so that $1-t= \pm \frac{r_{2}}{r_{2}-r_{1}}$.

$$
\left(r_{2}-r_{1}\right)^{2}(1-t)^{2}=r_{2}^{2}
$$

Now if $t=\frac{r_{1}}{r_{2}-r_{1}}$ then $1-t=1-\frac{r_{1}}{r_{2}-r_{1}}=\frac{1}{r_{2}-r_{1}}\left(r_{2}-2 r_{1}\right)$ and for this to equal $\pm \frac{r_{2}}{r_{2}-r_{1}}$ we would require either $r_{2}-2 r_{1}=r_{2}$ which would imply $r_{1}=0$; or $r_{2}-2 r_{1}=-r_{2}$ which would imply $r_{1}=r_{2}$ which is not allowed. It follows that $t=-\frac{r_{1}}{r_{2}-r_{1}}$ so that $1-t=1+\frac{r_{1}}{r_{2}-r_{1}}=\frac{r_{2}}{r_{2}-r_{1}}$.

\section{A cyclographic proof of the Feuerbach theorem}

We now show that the Incentre story we have developed so far fits into this cyclographic point of view. Recall the four incentres

$$
\begin{aligned}
& I_{0}=[1: 0: 0] \\
& I_{1}=\left[\left(1+t_{1} t_{2}\right)\left(1+t_{1} t_{3}\right):\left(1+t_{1}^{2}\right)\left(1-t_{2} t_{3}\right):\left(1+t_{1}^{2}\right)\left(t_{2}+t_{3}\right)\right] \\
& I_{2}=\left[\left(1+t_{1} t_{2}\right)\left(1+t_{2} t_{3}\right):\left(1+t_{2}^{2}\right)\left(1-t_{1} t_{3}\right):\left(1+t_{2}^{2}\right)\left(t_{1}+t_{3}\right)\right] \\
& I_{3}=\left[\left(1+t_{1} t_{3}\right)\left(1+t_{2} t_{3}\right):\left(1+t_{3}^{2}\right)\left(1-t_{1} t_{2}\right):\left(1+t_{3}^{2}\right)\left(t_{1}+t_{2}\right)\right] .
\end{aligned}
$$

And now introduce associated oriented_radii, which are

$r_{0} \equiv 1$

$r_{1} \equiv \frac{\left(t_{1}-t_{2}\right)\left(t_{1}-t_{3}\right)}{\left(1+t_{1} t_{2}\right)\left(1+t_{1} t_{3}\right)}$

$r_{2} \equiv \frac{\left(t_{2}-t_{3}\right)\left(t_{2}-t_{1}\right)}{\left(1+t_{1} t_{2}\right)\left(1+t_{2} t_{3}\right)}$

$r_{3} \equiv \frac{\left(t_{3}-t_{1}\right)\left(t_{3}-t_{2}\right)}{\left(1+t_{1} t_{3}\right)\left(1+t_{2} t_{3}\right)}$.

We have made some deliberate choices here. The ninepoint centre is

$N \equiv\left[x_{N}, y_{N}\right]=\left[8 w: u^{2}-v^{2}: 2 u v\right]$

where

$v=t_{1}+t_{2}+t_{3}+3 t_{1} t_{2} t_{3}, \quad u=t_{1} t_{2}+t_{1} t_{3}+t_{2} t_{3}+3$ and

$w=\left(t_{2} t_{3}+1\right)\left(t_{1} t_{3}+1\right)\left(t_{1} t_{2}+1\right)$.

Introduce its associated oriented_radius (noting the negative sign):

$$
\begin{aligned}
r_{N} & \equiv-\frac{\left(t_{1}^{2}+1\right)\left(t_{2}^{2}+1\right)\left(t_{3}^{2}+1\right)}{8\left(t_{1} t_{2}+1\right)\left(t_{1} t_{3}+1\right)\left(t_{2} t_{3}+1\right)} \\
& =-\frac{\left(t_{1}^{2}+1\right)\left(t_{2}^{2}+1\right)\left(t_{3}^{2}+1\right)}{8 w}=1-\frac{v^{2}+u^{2}}{8 w} .
\end{aligned}
$$

We have here used the identity

$u^{2}+v^{2}=$

$\left(t_{1}^{2}+1\right)\left(t_{2}^{2}+1\right)\left(t_{3}^{2}+1\right)+8\left(t_{2} t_{3}+1\right)\left(t_{1} t_{3}+1\right)\left(t_{1} t_{2}+1\right)$.

Now we are able to both state and prove Feuerbach's theorem in both a stronger and a more general setting: valid over a general field, and with an oriented aspect.

Theorem 7 (Oriented Feuerbach theorem) The oriented_circle $C_{N} \equiv\left[N, r_{N}\right]$ is tangent to each of the oriented_circles $C_{0} \equiv\left[I_{0}, r_{0}\right], C_{1} \equiv\left[I_{1}, r_{1}\right], C_{2} \equiv\left[I_{2}, r_{2}\right]$ and $C_{3} \equiv\left[I_{3}, r_{3}\right]$.

Proof. We first establish the easier tangency of $C_{N}$ and $C_{0}$ by first introducing the coordinates of $N$ and $r_{N}$ as above as

$N=\left[x_{N}, y_{N}\right]=\left[\frac{u^{2}-v^{2}}{8 w}, \frac{u v}{4 w}\right]$ and $r_{N}=1-\frac{u^{2}+v^{2}}{8 w}$.

Then the cyclographic condition for tangency of $C_{N}$ and $C_{0}$ is $x_{N}^{2}+y_{N}^{2}-\left(r_{N}-1\right)^{2}=0$ which becomes

$$
\left(\frac{u^{2}-v^{2}}{8 w}\right)^{2}+\left(\frac{2 u v}{8 w}\right)^{2}=\left(\frac{v^{2}+u^{2}}{8 w}\right)^{2}
$$

which is automatic. 
Now let's consider the more challenging tangency of $C_{N}$ and $C_{1}$, which amounts to the condition

$$
\begin{aligned}
& \left(x_{N}-\frac{\left(1+t_{1}^{2}\right)\left(1-t_{2} t_{3}\right)}{\left(1+t_{1} t_{2}\right)\left(1+t_{1} t_{3}\right)}\right)^{2}+\left(y_{N}-\frac{\left(1+t_{1}^{2}\right)\left(t_{2}+t_{3}\right)}{\left(1+t_{1} t_{2}\right)\left(1+t_{1} t_{3}\right)}\right)^{2} \\
& =\left(r_{N}-\frac{\left(t_{1}-t_{2}\right)\left(t_{1}-t_{3}\right)}{\left(1+t_{1} t_{2}\right)\left(1+t_{1} t_{3}\right)}\right)^{2}
\end{aligned}
$$

which we can write more concisely as

$$
\begin{aligned}
& \left(\frac{u^{2}-v^{2}}{8 w}-\frac{\left(1+t_{1}^{2}\right)\left(1-t_{2}^{2} t_{3}^{2}\right)}{w}\right)^{2} \\
& +\left(\frac{2 u v}{8 w}-\frac{\left(1+t_{1}^{2}\right)\left(t_{2}+t_{3}\right)\left(1+t_{2} t_{3}\right)}{w}\right)^{2} \\
& =\left(1-\frac{u^{2}+v^{2}}{8 w}-\frac{\left(t_{1}-t_{2}\right)\left(t_{1}-t_{3}\right)\left(1+t_{2} t_{3}\right)}{w}\right)^{2} .
\end{aligned}
$$

Now after establishing common denominators, the numerators of the terms in this equation will be

$$
\begin{aligned}
A \equiv & u^{2}-v^{2}-8\left(1+t_{1}^{2}\right)\left(1-t_{2}^{2} t_{3}^{2}\right) \\
= & -t_{1}^{2} t_{2}^{2} t_{3}^{2}+t_{1}^{2} t_{2}^{2}+t_{1}^{2} t_{3}^{2}+9 t_{2}^{2} t_{3}^{2}-4 t_{1}^{2} t_{2} t_{3}-4 t_{1} t_{2}^{2} t_{3} \\
& -4 t_{1} t_{2} t_{3}^{2}+4 t_{1} t_{2}+4 t_{1} t_{3}+4 t_{2} t_{3}-9 t_{1}^{2}-t_{2}^{2}-t_{3}^{2}+1 \\
B \equiv & 2 u v-8\left(1+t_{1}^{2}\right)\left(t_{2}+t_{3}\right)\left(1+t_{2} t_{3}\right) \\
= & -2 t_{1}^{2} t_{2}^{2} t_{3}-2 t_{1}^{2} t_{2} t_{3}^{2}+6 t_{1} t_{2}^{2} t_{3}^{2}-6 t_{1}^{2} t_{3}-6 t_{2}^{2} t_{3}-6 t_{2} t_{3}^{2} \\
& -6 t_{1}^{2} t_{2}+2 t_{1} t_{2}^{2}+2 t_{1} t_{3}^{2}+24 t_{1} t_{2} t_{3}+6 t_{1}-2 t_{2}-2 t_{3}
\end{aligned}
$$

and

$$
\begin{aligned}
C \equiv & 8 w-\left(u^{2}+v^{2}\right)-8\left(t_{1}-t_{2}\right)\left(t_{1}-t_{3}\right)\left(1+t_{2} t_{3}\right) \\
= & -t_{1}^{2} t_{2}^{2} t_{3}^{2}-t_{1}^{2} t_{2}^{2}-t_{1}^{2} t_{3}^{2}-9 t_{2}^{2} t_{3}^{2}-8 t_{1}^{2} t_{2} t_{3}+8 t_{1} t_{2}^{2} t_{3} \\
& +8 t_{1} t_{2} t_{3}^{2}+8 t_{1} t_{2}+8 t_{1} t_{3}-8 t_{2} t_{3}-9 t_{1}^{2}-t_{2}^{2}-t_{3}^{2}-1
\end{aligned}
$$

and then $A^{2}+B^{2}=C^{2}$ is a valid Pythagorean identity in $t_{1}, t_{2}$ and $t_{3}$. This establishes the tangency of $C_{N}$ and $C_{1}$, and the cases of $C_{N}$ and $C_{2}$, and $C_{N}$ and $C_{3}$, follow symmetrically.

Feuerbach's theorem is then a consequence because we know tangency of oriented_circles implies tangency of the associated circles. Note that our proof extends the oriented version, which is more powerful than the original, to general fields.

We may now well ask: what is the geometric meaning of "oriented_circles" over more general fields, for example the complex numbers, or a finite field? We do not have a good answer to this interesting question at this point. It appears that our understanding of universal geometry is still in early stages!

\section{Points of tangency}

We can now use the Oriented tangency theorem to find the points of tangency of the incircles with the nine-point circle to be:

$$
\begin{aligned}
J_{0} & \equiv C_{0} C_{N}=\frac{r_{N}\left[x_{0}, y_{0}\right]-r_{0}\left[x_{N}, y_{N}\right]}{r_{N}-r_{0}} \\
& =\left[r_{0}-r_{N}: r_{0} x_{N}-x_{0} r_{N}: r_{0} y_{N}-y_{0} r_{N}\right] \\
J_{1} & \equiv C_{1} C_{N}=\frac{r_{N}\left[x_{1}, y_{1}\right]-r_{1}\left[x_{N}, y_{N}\right]}{r_{N}-r_{1}} \\
& =\left[r_{1}-r_{N}: r_{1} x_{N}-x_{1} r_{N}: r_{1} y_{N}-y_{1} r_{N}\right] \\
J_{2} & \equiv C_{2} C_{N}=\frac{r_{N}\left[x_{2}, y_{2}\right]-r_{2}\left[x_{N}, y_{N}\right]}{r_{N}-r_{2}} \\
& =\left[r_{2}-r_{N}: r_{2} x_{N}-x_{2} r_{N}: r_{2} y_{N}-y_{2} r_{N}\right] \\
J_{3} & \equiv C_{3} C_{N}=\frac{r_{N}\left[x_{3}, y_{3}\right]-r_{3}\left[x_{N}, y_{N}\right]}{r_{N}-r_{3}} \\
& =\left[r_{3}-r_{N}: r_{3} x_{N}-x_{3} r_{N}: r_{3} y_{N}-y_{3} r_{N}\right] .
\end{aligned}
$$

\section{Additional features in finite fields}

Our geometrical intuition has been directed for thousands of years by physical constructions on flat surfaces. But with the view of Rational trigonometry, we see that metrical theorems can be investigated even over finite fields. The formulas that we have so far derived In our set-up of the Feuerbach theorem are applicable to arbitrary fields, but there are additional aspects that appear which we can illustrate in the simple case of a finite prime field $\mathbb{F}_{p}$.

We assumed that the three original $t$ values that determined the original points on the unit circle were distinct, that no $t$ value satisfies $t^{2}=-1$, and that the product of any two $t$ values is not equal to -1 . In addition we usually separate the case where the oriented radius of the nine-point circle is equal to the oriented_radius of any of the incircles, for example if we start with an equilateral triangle.

It is of some interest to investigate cases over a finite field when such assumptions are not necessarily holding. This leads to situations where aspects of the Feuerbach framework hold, but others do not which will be unfamiliar to the student of Euclidean geometry over the real or rational numbers. Finite field geometry is a rich ground which holds the promise to enrich the subject and strengthen ties to number theory and combinatorics.

To focus the discussion, we consider the case of working in the field $\mathbb{F}_{17}$. When one of our formulas does not apply, because a denominator in the affine expression is 0 , we substitute a blank -. 


\section{Examples in $\mathbb{F}_{17}$}

\subsection{Example 1: $t$-values of $\{3,7,15\}$}

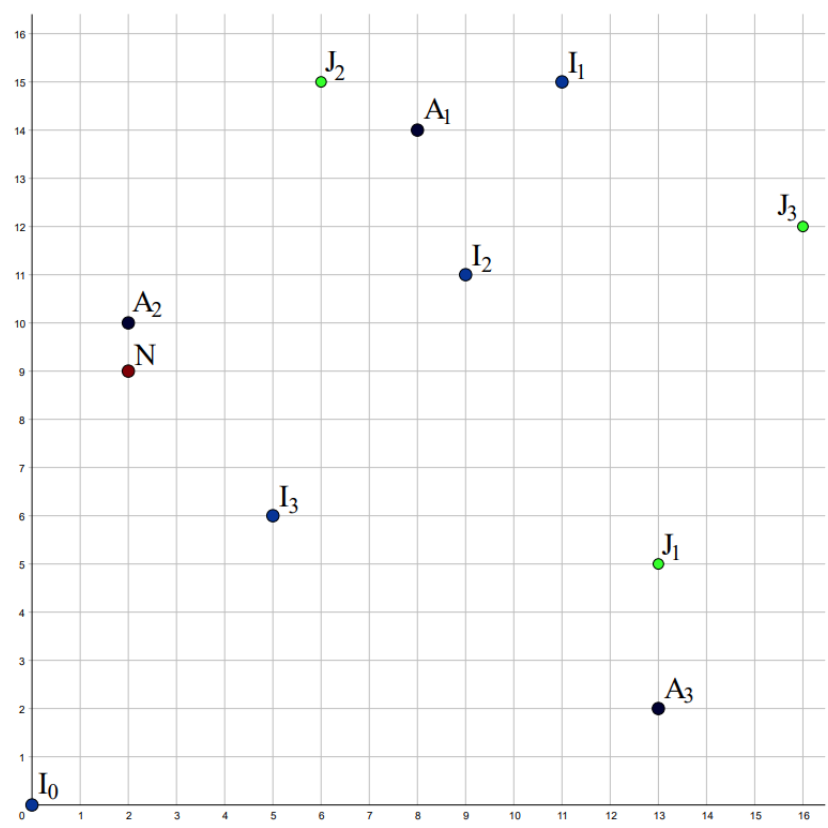

Figure 9: Example 1 with $t$ values of $3,7,15$ in $\mathbb{F}_{17}$

With these $t$-values, letting $t_{1}=3, t_{2}=7$ and $t_{3}=15$, we obtain the following:

$$
\begin{array}{llll} 
& I_{0}=[0,0] & r_{0}=1 & \\
A_{1}=[8,14] & I_{1}=[11,15] & r_{1}=11 & J_{0}=- \\
A_{2}=[2,10] & I_{2}=[9,11] & r_{2}=12 & J_{1}=[13,5] \\
A_{3}=[13,2] & I_{3}=[5,6] & r_{3}=2 & J_{2}=[6,15] \\
& N=[2,9] & r_{N}=1 & J_{3}=[16,12]
\end{array}
$$

Since $r_{0}=r_{N}$, the expressions for the coordinates of the corresponding point of tangency $J_{0}$ will be undefined.

Remark 5 Such a case does not arise when working in the rational field. This is because only one of the incircles has a radii with the same sign as that of the nine-point circle, and that incircle can only have the same radius as the nine-point circle if their centres coincide (which only occurs when the starting triangle is equilateral).

\subsection{Example 2: $t$-values of $\{2,7,11\}$}

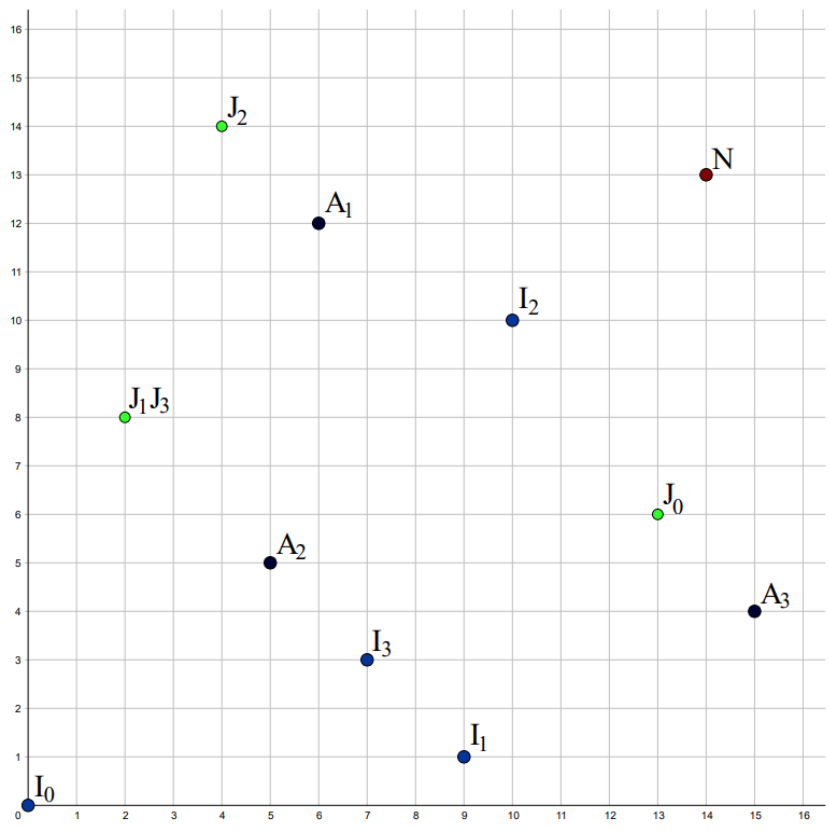

Figure 10: Example 2 with $t$ values of $2,7,11$ in $\mathbb{F}_{17}$

With these $t$-values, letting $t_{1}=2, t_{2}=7$ and $t_{3}=11$, we obtain the following:

$$
\begin{array}{llll} 
& I_{0}=[0,0] & r_{0}=1 & \\
A_{1}=[6,12] & I_{1}=[9,1] & r_{1}=9 & J_{0}=[13,6] \\
A_{2}=[5,5] & I_{2}=[10,10] & r_{2}=1 & J_{1}=[2,8] \\
A_{3}=[15,4] & I_{3}=[7,3] & r_{3}=4 & J_{2}=[4,14] \\
& N=[14,13] & r_{N}=13 & J_{3}=[2,8]
\end{array}
$$

Since the radius of the nine-point circle is not the same as the radius of any of the incircles this time, none of the $t$-values are a square root of -1 and no product of two $t$ values is -1 , we can show that the nine-point circle is tangent to the four incircles of the triangle $\overline{A_{1} A_{2} A_{3}}$. However, we do run into the issue of $J_{1}$ and $J_{3}$ coinciding.

Remark 6 While a proof is not attempted here, it is suspected that there does not exist a case where all the tangent points exist and are distinct in $\mathbb{F}_{17}$, based on extensive (but not exhaustive) testing of different combinations of $t$-values in $\mathbb{F}_{17}$. This is most likely a result of $\mathbb{F}_{17}$ being "too small", for all J points to be distinct. 


\subsection{Example 3: $t$-values of $\{4,7,15\}$}

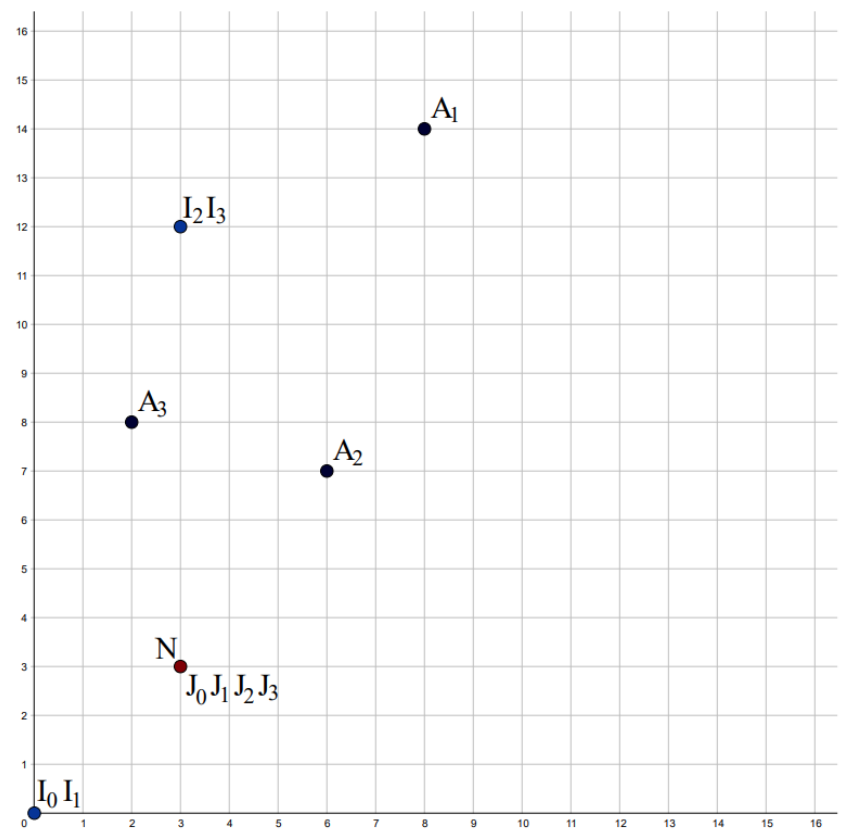

Figure 11: Example 3 with $t$ values of $4,7,15$ in $\mathbb{F}_{17}$

With these $t$-values, we have exactly one square root of -1 and no products for -1 . Letting $t_{1}=4, t_{2}=7$ and $t_{3}=15$, we obtain the following:

$$
\begin{array}{llll} 
& I_{0}=[0,0] & r_{0}=1 & \\
A_{1}=[8,14] & I_{1}=[0,0] & r_{1}=16 & J_{0}=[3,3] \\
A_{2}=[6,7] & I_{2}=[3,12] & r_{2}=8 & J_{1}=[3,3] \\
A_{3}=[2,8] & I_{3}=[3,12] & r_{3}=9 & J_{2}=[3,3] \\
& N=[3,3] & r_{N}=0 & J_{3}=[3,3]
\end{array}
$$

As expected, since we have a square root of -1 in our $t$ values, we get overlapping incentres and a nine-point radius of zero, resulting in all the points of tangency concentrating at the nine-point centre. These overlapping coordinates are a result of the factor $\left(1+t_{1}^{2}\right)$ going to zero since $t_{1}$ is a square root of -1 in our field. This means that $I_{1}$ has both coordinates go to zero while $I_{2}$ and $I_{3}$ simplify to get the same coordinates. This case results in the ninepoint circle becoming a null_circle. Notably, since we do not have any products of $t$-values giving -1 , we are still able to find coordinates for all the significant points.

\section{A complete finite field example in $\mathbb{F}_{23}$}

If we choose our $t$-values in $\mathbb{F}_{23}$ to be $t_{1}=2, t_{2}=5$ and $t_{3}=13$, we obtain the following:

$$
\begin{array}{llll} 
& I_{0}=[0,0] & r_{0}=1 & \\
A_{1}=[6,17] & I_{1}=[22,1] & r_{1}=18 & J_{0}=[10,4] \\
A_{2}=[11,21] & I_{2}=[19,7] & r_{2}=7 & J_{1}=[3,4] \\
A_{3}=[18,9] & I_{3}=[1,12] & r_{3}=8 & J_{2}=[1,6] \\
& N=[18,21] & r_{N}=13 & J_{3}=[6,16]
\end{array}
$$

Since we can find a nice example in $\mathbb{F}_{23}$ without any significant points overlapping, we know that the "smallest" prime finite field for the Feuerbach theorem (which we can think of as the finite field of least order such that all significant points can be distinct with an appropriate choice of $t$-values) will be less that or equal to $\mathbb{F}_{23}$. It is perhaps interesting to consider this kind of question, which has both a geometrical, a combinatorial, and a number theoretic aspect simultaneously.

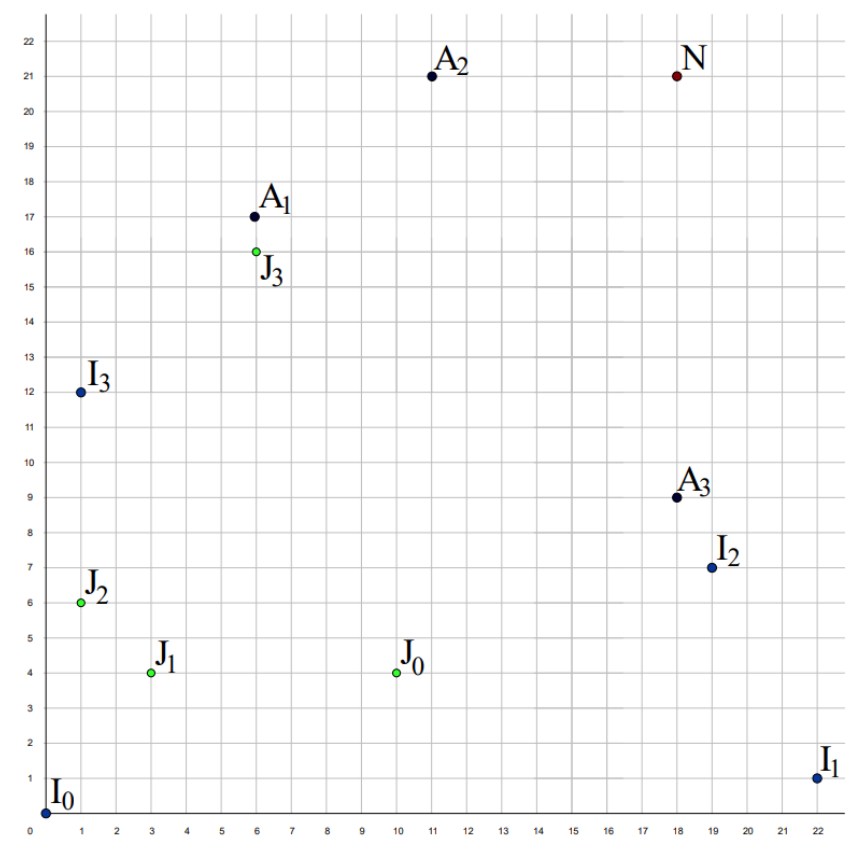

Figure 12: When $t_{1}=2, t_{2}=5$ and $t_{3}=13$, we get the following constructions in $\mathbb{F}_{23}^{2}$

\section{References}

[1] J. CASEY, On the Equations and Properties: (1) of the System of Circles Touching Three Circles in a Plane; (2) of the System of Spheres Touching Four Spheres in Space; (3) of the System of Circles Touching Three Circles on a Sphere; (4) of the System of Conics Inscribed to a Conic, and Touching Three Inscribed Conics in a Plane, Proceedings of the Royal Irish Academy 9 (1864), 396-423.

[2] H. S. M. Coxeter, S. L. Greitzer, Geometry Revisited, Math. Assoc. Amer., 1967, 117-119. 
[3] K. W. FEUERBACH, Eigenschaften einiger merkwürdigen Punkte des geradlinigen Dreiecks, und mehrerer durch die bestimmten Linien und Figuren, Nürnberg, Germany: Riegel und Wiesner, 1822.

[4] F. Hofbauer, A simple proof of Feuerbach's theorem, https://arxiv.org/abs/1610.03962, 2016.

[5] R. Lachlan, An Elementary Treatise on Modern Pure Geometry, London: Macmillan, 1893, 70-71.

[6] N. LE, N. J. WILDBERGER, Incenter Circles, Chromogeometry, and the Omega Triangle, KoG 18 (2014), 5-18.

\section{William Beare}

e-mail: w.beare@student.unsw.edu.au

School of Mathematics and Statistics UNSW

Sydney, Australia
[7] W. J. M'Clelland, A Treatise on the Geometry of the Circle and Some Extensions to Conic Sections by the Method of Reciprocation, with Numerous Examples, London: Macmillan, 1891, 225-226.

[8] M. J. G. ScheER, A Simple Vector Proof of Feuerbach's Theorem, Forum Geom. 11 (2011), 205-210.

[9] N. J. WILDBERGER, Divine proportions: Rational Trigonometry to Universal Geometry, Wild Egg Books, 2005.

[10] N. J. WildBerger, Affine and Projective Universal Geometry, http://arxiv.org/abs/math/ 0612499v1, 2006.

\author{
N. J. Wildberger \\ orcid.org/0000-0003-3503-6495 \\ e-mail: n.wildberger@unsw.edu.au \\ School of Mathematics and Statistics UNSW \\ Sydney, Australia
}

